\title{
A Comparison Study of Four Cervical Disk Arthroplasty Devices Using Finite Element Models
}

\author{
Yuvaraj Purushothaman ${ }^{1,2}$, Hoon Choi ${ }^{1}$, Narayan Yoganandan ${ }^{1}$, \\ Davidson Jebaseelan ${ }^{2}$, Jamie Baisden ${ }^{1}$, Shekar Kurpad ${ }^{1}$ \\ ${ }^{I}$ Center for NeuroTrauma Research, Department of Neurosurgery, Medical College of Wisconsin, Milwaukee, WI, USA \\ ${ }^{2}$ School of Mechanical Engineering, Vellore Institute of Technology (Chennai Campus), Chennai, India
}

Study Design: The study examined and compared four artificial cervical disks using validated finite element models.

Purpose: To compare and contrast the biomechanical behavior of four artificial cervical disks by determining the external (range of motion) and internal (facet force and intradiscal pressure) responses following cervical disc arthroplasty (CDA) and to elucidate any device design effects on cervical biomechanics.

Overview of Literature: Despite CDA's increasing popularity most studies compare the CDA procedure with anterior cervical discectomy and fusion. There is little comparative evaluation of different artificial disks and, therefore, little understanding of how varying disk designs may influence spinal biomechanics.

Methods: A validated C2-T1 finite element model was subjected to flexion-extension. CDAs were simulated at the C5-C6 level with the Secure-C, Mobi-C, Prestige LP, and Prodisc $\mathrm{C}$ prosthetic disks. We used a hybrid loading protocol to apply sagittal moments. Normalized motions at the index and adjacent levels, and intradiscal pressures and facet column loads were also obtained.

Results: The ranges of motion at the index level increased after CDA. The Mobi-C prosthesis demonstrated the highest amount of flexion, followed by the Secure-C, Prestige LP, and Prodisc C. The Secure-C demonstrated the highest amount of extension, followed by the Mobi-C, Prodisc C, and Prestige LP. The motion decreased at the rostral and caudal adjacent levels. Facet forces increased at the index level and decreased at the rostral and caudal adjacent levels following CDA. Intradiscal pressures decreased at the adjacent levels for the Mobi-C, Secure-C, and Prodisc C. Conversely, the use of the Prestige LP increased intradiscal pressure at both adjacent levels.

Conclusions: While all artificial disks were useful in restoring the index level motion, the Secure-C and Mobi-C translating abilities allowed for lower intradiscal pressures at the adjacent segments and may be the driving mechanism for minimizing adjacent segment degenerative arthritic changes. The facet joint integrity should also be considered in the clinical decision-making process for CDA selection.

Keywords: Adjacent level motions; Angular kinematics; Total disc replacement; Discectomy; Index level biomechanics; Finite element model

Received Mar 22, 2020; Revised May 18, 2020; Accepted Jun 9, 2020

Corresponding author: Narayan Yoganandan

Center for NeuroTrauma Research, Department of Neurosurgery, Medical College of Wisconsin, 8701 Watertown Plank Road, Milwaukee, WI 53226, USA

Tel: +1-414-333-3269, Fax: +1-414-955-9201, E-mail: yoga@mcw.edu 


\section{Introduction}

For many years, anterior cervical discectomy and fusion (ACDF) has been a conventional surgical treatment for symptomatic cervical spondylosis [1]. While the motion at the index level is constrained due to fusion, fusion surgery's effect on the adjacent vertebral levels remains unclear [2-4]. Cervical disk arthroplasty (CDA), a more recent procedure approved by the Food and Drug Administration (FDA) is aimed at preserving motion at the index level while minimizing the adjacent segment degeneration (ASD). Over the past 13 years, the FDA has approved several cervical prosthetic disk devices [5-7]. While the intent is to preserve the motion and minimize ASD, the design features and material properties vary among the arthroplasty implants. Clinical studies, such as those cited above, have provided information on effectiveness, depending on the outcomes, and their length of outcomes in different populations. In general, all FDA approved devices are acceptable alternatives to the ACDF. However, issues such as heterotopic ossification, the extent of motion preservation, and adjacent segment biomechanics vary among the devices. The present study's objective was to determine motions, intradiscal pressures, and facet forces representing the external intervertebral joint-specific kinematics, and internal anterior and posterior column load-sharing of four FDA approved CDAs using finite element models.

\section{Methods}

\section{Intact spinal column}

We used a validated finite element model of the C2-T1 portion of the spinal column $[8,9]$. The model included the following components: (1) cancellous core and cortical shell of the vertebral bodies, their posterior elements, i.e., laminae, pedicles and spinous processes, and the dens of the axis; (2) intervertebral disk annular fibers, ground substance, and nucleus pulposus; and (3) anterior and posterior longitudinal ligaments, joint capsules of the lateral mass, ligamentum flavum, and interspinous ligaments. The seven segments meshed with hexahedral elements, and material properties were obtained from the literature [10-12]. The disk anterior and posterior regions consisted of 16 and eight layers [13]. We used 11,452 elements in the model. The hyper-elastic foam ground substance was defined using the Hill strain energy function. The fibers were defined using membrane elements with tension-only directional fibers embedded in the ground substance. The fibers in the anterior annulus were defined in a crisscross manner, while the fibers in the posterior region were defined in the vertical direction [14]. Fig. 1 shows the coronal view of the intact spinal column. Table 1 shows the material properties used in the intact and four CDA spine models. Appendix 1 includes definitions of some terminologies.

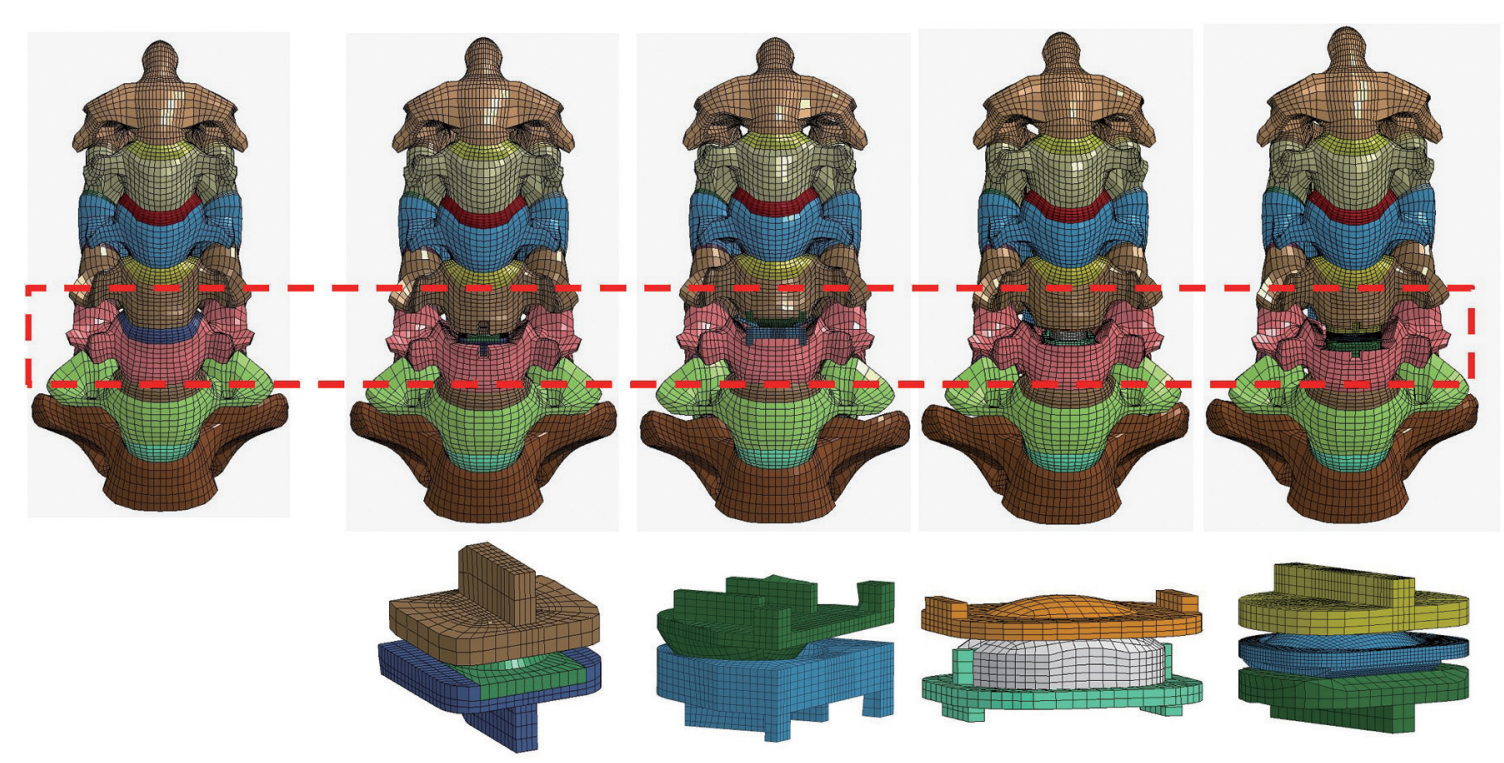

Fig. 1. Coronal views from left to right of intact, Prodisc C, Prestige LP, Mobi-C, and Secure-C and spine models. Dashed rectangle shows the level of cervical disk arthroplasties, i.e., at the C5-C6 level. 


\section{Artificial disks}

The Secure-C CDA manufactured by Globus Medical Inc. (Audubon, PA, USA), Mobi-C, manufactured by Zimmer Biomet (Warsaw, IN, USA), Prodisc C manufactured by Centinel Spine (West Chester, PA, USA), and Prestige LP manufactured by Medtronic Sofamor Danek (Memphis, TN, USA) were used in the study for single-level surgery [15]. Ultra-high-molecular-weight polyethylene (UHMWPE) material is used in the first three devices; however, in the Prestige LP, only metal components are used.

\section{1) Prodisc $C$}

This is a two-piece device with metal (cobalt chromium alloy) on polyethylene; the FDA approved this device for human use in 2007. It allows for flexion, extension, and lateral bending, up to $20^{\circ}$ of angulation in each mode, with unlimited axial rotation. It has a fixed center of rotation. The immediate endplate fixation at the end surfaces of the device is via a midline keel. The coupled motion is such that the translation is allowed only when combined with rotation.

\section{2) Prestige LP}

This is also a two-piece device, but it is metal-on-metal (titanium alloy). The FDA approved this device in 2014. It allows for more than $10^{\circ}$ of angulation, each in flexion, extension, and lateral bending, with unlimited lateral rotation. The allowable anterior/posterior translation is 2 $\mathrm{mm}$. While there is no fixed center of rotation, it lies at the center of the inverted dome affixed to the superior section of the device. The immediate endplate fixation at the end surfaces utilizes bone screws and a locking mechanism.

Geometry: The geometry of the Prodisc C and Prestige LP was modeled using the actual devices, and their material properties were obtained from the literature. The implant size selection and placement in the vertebral segments were simulated according to the standard surgical

Table 1. Material Properties used in the model for the intact spine components and cervical disk arthroplasties

\begin{tabular}{|c|c|c|c|}
\hline Component & Element type & Constitutive model & Parameters \\
\hline Cortical bone & Quadrilateral shell & Linear elastic & $\mathrm{E}=16.8 \mathrm{GPa}, \mu=0.3$ \\
\hline Trabecular bone & Hexahedral solid & Linear elastic & $\mathrm{E}=0.4 \mathrm{GPa}, \mu=0.3$ \\
\hline Endplate & Quadrilateral shell & Linear elastic & $\mathrm{E}=5.6 \mathrm{GPa}, \mu=0.3$ \\
\hline Facet cartilage & Quadrilateral shell & Linear elastic & $\mathrm{E}=0.01 \mathrm{GPa}, \mu=0.3$ \\
\hline Annulus ground substance & Hexahedral solid & Hill foam & $\mathrm{n}=2, \mathrm{C} 1=0.000115 \mathrm{GPa}, \mathrm{C} 2=0.002101 \mathrm{GPa}, \mathrm{C} 3=-0.000893 \mathrm{GPa}, \mathrm{b} 1=-2, \mathrm{~b} 2=-1, \mathrm{~b} 3=-2$ \\
\hline Annulus fibrosus & Quadrilateral membrane & Orthotropic nonlinear elastic & Fiber angle $\left(45^{\circ}-60^{\circ}\right)$ \\
\hline Nucleus pulposus & Hexahedral solid & Fluid & $\mathrm{K}=1.720 \mathrm{GPa}$ \\
\hline Ligaments & Quadrilateral membrane & Non-linear curves & Stress-strain curves \\
\hline \multicolumn{4}{|l|}{ Prodisc C } \\
\hline Upper plate & Hexahedral solid & Linear elastic & $\mathrm{E}=210 \mathrm{GPa}, \mu=0.3$ \\
\hline Middle core & Hexahedral solid & Linear elastic & $E=3 \mathrm{GPa}, \mu=0.3$ \\
\hline Lower plate & Hexahedral solid & Linear elastic & $\mathrm{E}=210 \mathrm{GPa}, \mu=0.3$ \\
\hline \multicolumn{4}{|l|}{ Prestige LP } \\
\hline Upper plate & Hexahedral solid & Linear elastic & $E=110 \mathrm{GPa}, \mu=0.3$ \\
\hline Lower plate & Hexahedral solid & Linear elastic & $E=110 \mathrm{GPa}, \mu=0.3$ \\
\hline \multicolumn{4}{|l|}{ Mobi-C } \\
\hline Upper plate & Hexahedral solid & Linear elastic & $\mathrm{E}=210 \mathrm{GPa}, \mu=0.3$ \\
\hline Middle core & Hexahedral solid & Linear elastic & $E=3 \mathrm{GPa}, \mu=0.3$ \\
\hline Lower plate & Hexahedral solid & Linear elastic & $\mathrm{E}=210 \mathrm{GPa}, \mu=0.3$ \\
\hline \multicolumn{4}{|l|}{ Secure-C } \\
\hline Upper plate & Hexahedral solid & Linear elastic & $\mathrm{E}=210 \mathrm{GPa}, \mu=0.3$ \\
\hline Middle core & Hexahedral solid & Linear elastic & $E=3 \mathrm{GPa}, \mu=0.3$ \\
\hline Lower plate & Hexahedral solid & Linear elastic & $\mathrm{E}=210 \mathrm{GPa}, \mu=0.3$ \\
\hline
\end{tabular}


techniques. The Prodisc C model consisted of two cobalt chrome alloy endplates and a core made of UHMWPE (Fig. 1). The Prestige LP consisted of two titanium carbide endplates, and the inverted dome of the superior endplate articulated with the groove of the inferior endplate [16].

\section{3) Mobi-C}

This three-piece device is comprised of metal on a polyethylene mobile core. The metal portion is made of a cobalt chromium alloy. The FDA approved this device for human use in 2013. It allows for more than $10^{\circ}$ of angulation in each flexion, extension, and lateral bending, with unlimited lateral rotation. The allowable anterior/posterior translation is $1 \mathrm{~mm}$. While there is no fixed center of rotation, it moves in the lateral and anteroposterior directions, depending on the core position. The immediate endplate fixation at the end surfaces is via inclined teeth for purchase into the vertebral body.

\section{4) Secure-C}

This is a three-piece device with metal on polyethylene (a sliding core with spherical superior and cylindrical inferior cores). It was approved for human use by the FDA in 2012. The metal portion consists of a cobalt chromium alloy. It allows for $30^{\circ}$ of angulation in both flexion and extension, $20^{\circ}$ in lateral bending and $\pm 1.25 \mathrm{~mm}$ translation in the sagittal plane. There is no fixed center of rotation. The immediate endplate fixation at the end surfaces is via multiple serrated keel for purchase into the vertebral body.

Geometry: The geometry of the Secure- $\mathrm{C}$ and Mobi-C was modeled as follows: A coordinate Measuring Machine (FaroArm, Irvine, CA, USA) was used to define the geometric representation of objects using dense clouds of three-dimensional points from the external surface of the disk prosthesis. The generated three-dimensional points were imported into a software (CATIA V6; Dassault Systems, Waltham, MA, USA) and processed with a digitized shape editor to create the surface geometry from the points and meshed in the ANSA software (BETA CAE Systems, Farmington Hills, MI, USA).

\section{Surgical simulations}

Standard surgical procedures were used to simulate all four devices at the C5-C6 motion segment. The anterior longitudinal ligament was removed at the index level, and a cavity was created to place the disk prosthesis. Both the superior and inferior components of the devices were attached to the respective vertebral bodies using tied contacts to simulate complete osteointegration of the implant with the bone. This ensured no relative motion between the device and the vertebral endplates. For the Prodisc C, Secure- $\mathrm{C}$, and Mobi-C devices, the contact between the metal and polymer surfaces were modeled as a surface to surface contact definition, with a coefficient of friction of 0.3. For the Prestige LP, the surface to surface contact definition was maintained between the two metal surfaces, and the coefficient of friction was set at 0.1. Fig. 1 includes the coronal views of the spine implanted with the all CDAs.

\section{Load application}

Flexion and extension bending moments were applied to the spine to analyze the four CDAs. The procedure followed the conventional methods, i.e., use a pure moment loading to the intact spine, in both modalities. The pure moment load levels were $2 \mathrm{Nm}$ each in flexion and extension. We determined the overall range of motion (ROM) of the spinal column. In other words, the $\mathrm{C} 3-\mathrm{C} 7$ motion was obtained for the intact spine. Then, we determined the bending moment in both modes to each CDA spine that matches the ROM determined in the pure moment condition for the intact spine. This was accomplished by varying the applied moment until the overall column ROM of the CDA-introduced spine reached the magnitude determined in the intact spine under pure moment loading. The hybrid moment protocol was followed in this present study. The overall and segmental ROMs, intradiscal pressures, and facet forces were obtained. Continuously increasing combined loading may be a topic for future study [17]. These data from the CDA spines were used to compare the changes in the external and internal biomechanical responses of the spine by normalizing with respect to the intact spine using the following equation, where parameter refers to $\mathrm{ROM}$, intradiscal pressure, and facet loads for each CDA.

Normalized (parameter $)=100 \times \frac{(\text { CDA magnitude-intact spine magnitude })}{(\text { Intact spine magnitude })}$ 


\section{Results}

\section{Responses under pure moment}

In flexion, the $\mathrm{C} 5-\mathrm{C} 6$ and $\mathrm{C} 6-\mathrm{C} 7$ segments of the intact spine responded with the greatest angulations, $10.7^{\circ}$ and $10.2^{\circ}$, while the $\mathrm{C} 2-\mathrm{C} 3$ and $\mathrm{C} 7-\mathrm{T} 1$ responded with the lowest angulations, $8.0^{\circ}$ and $8.7^{\circ}$. In extension, the $\mathrm{C} 5-\mathrm{C} 6$ segment responded with the greatest angulation of $6.0^{\circ}$, and the $\mathrm{C} 2-\mathrm{C} 3$ segment responded with the least angulation of $4.3^{\circ}$. The overall column motion, defined as the summation of each segment's motion, was $56.6^{\circ}$ in flexion and $30.0^{\circ}$ in extension. Fig. 2 shows a bar chart of the range of segmental motions at each level for both sagittal bending moments.

\section{Moments and range of motion responses under hy- brid loading}

Sagittal bending moments required to achieve the overall motion of the intact spine for the Secure- $\mathrm{C}$, Mobi-C, Prodisc C, and Prestige LP in flexion were $1.8 \mathrm{Nm}, 1.9 \mathrm{Nm}$, $1.8 \mathrm{Nm}$, and $2.3 \mathrm{Nm}$, and in extension they were $1.3 \mathrm{Nm}$, $1.4 \mathrm{Nm}, 1.4 \mathrm{Nm}$, and $2.2 \mathrm{Nm}$, respectively. Fig. 3 illustrates changes in the motion responses of the four CDAs concerning the intact spine in flexion at the rostral, index, and caudal levels. The decreased normalized responses at the caudal segment were $11.6 \%$ and $11.5 \%$ for the Mobi-C and Prestige LP, while they were 5.9\% and $4.3 \%$ for the Prodisc $\mathrm{C}$ and Secure-C CDAs. At the rostral segment, decreases ranged from $5.4 \%$ for the Prestige LP to $7.2 \%$ for the Mobi- $\mathrm{C}$ disk. At the index level, Mobi- $\mathrm{C}$ increased the motion by $31.6 \%$, Secure-C by $5.6 \%$, and the Prestige LP and Prodisc C increased by $15.5 \%$ and $14.5 \%$, respectively.

Fig. 4 shows changes in the motion responses of the CDAs concerning the intact spine in extension at the three levels. At the rostral segment, motions decreased by $18.8 \%$ and $17.2 \%$ for the Prodisc $\mathrm{C}$ and Mobi-C, while they decreased by $15.7 \%$ and $12.5 \%$ for the Secure-C and Prestige LP disks. At the caudal segment, decreases ranged from 5.7\% for the Prestige LP to 7.1\% for the Prodisc C device. At the index level, Secure- $\mathrm{C}$ increased the motion by $48.1 \%$, Mobi-C by $36.5 \%$, and Prestige LP and Prodisc C increased by $26.5 \%$ and $35.5 \%$, respectively.

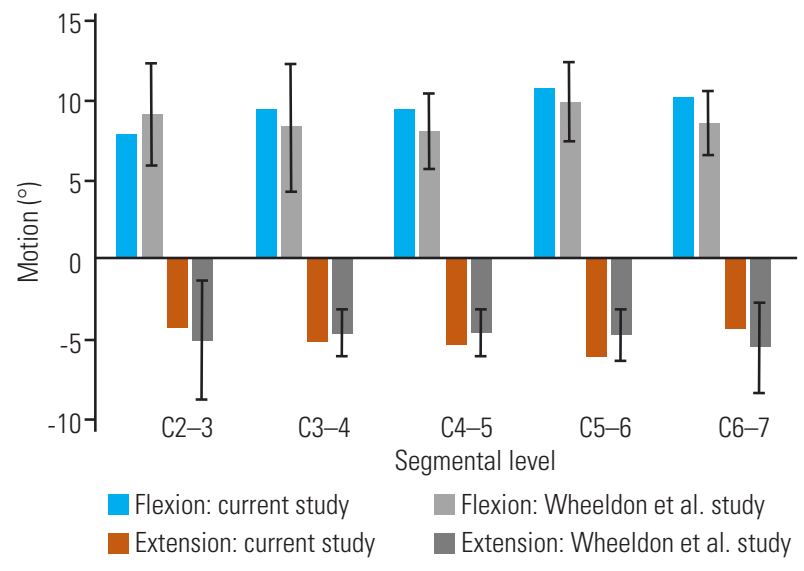

Fig. 2. Bar chart showing the segmental motions under flexion and extension for the intact spine along with standard deviations from experimental studies.



Fig. 3. Bar chart showing the normalized motions under flexion for different cervical disk arthroplasties.

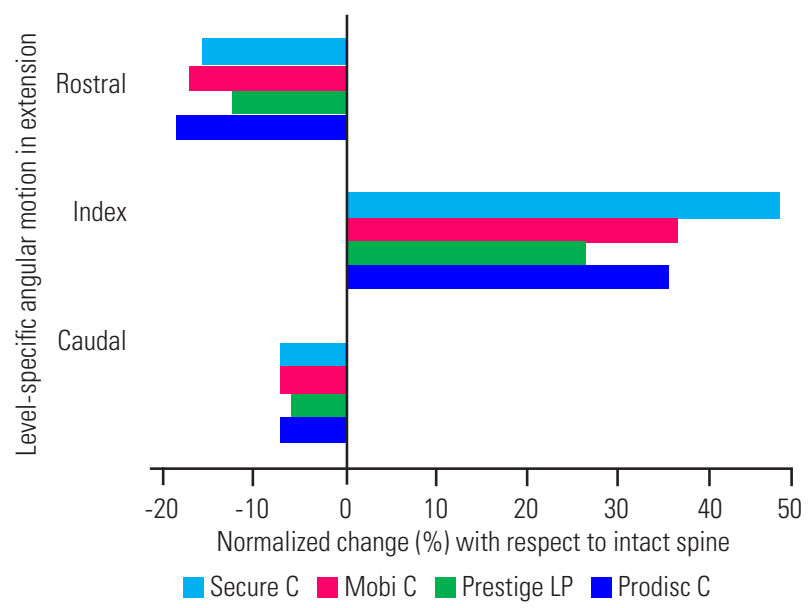

Fig. 4. Bar chart showing the normalized motions under extension for different cervical disk arthroplasties. 


\section{Intradiscal pressure responses under hybrid loading protocol}

Fig. 5 shows changes in the intradiscal pressure responses of the four CDAs concerning the intact spine in the flexion at adjacent levels. At the caudal segment, pressures decreased by $13.6 \%$ for the Mobi-C, $4.5 \%$ for the Secure-C and $3.3 \%$ for the Prodisc C, while it increased by $5.8 \%$ for the Prestige LP device. At the rostral segment, decreases ranged from $4.5 \%$ for the Mobi-C to 3.1\% for the SecureC to $1.9 \%$ for the Prodisc C, while for the Prestige LP, disk pressures increased by $5.7 \%$ compared to the intact spine.

Fig. 6 shows changes in the intradiscal pressure responses of the four CDAs with respect to the intact spine in extension at the adjacent levels. At the rostral segment, pressures decreased by $31.7 \%$ for the Mobi-C, $23.9 \%$ for the Secure-C and $19.0 \%$ for the Prodisc C, while it increased by $8.2 \%$ for the Prestige LP device. At the caudal segment, the decreases ranged from $22.0 \%$ for the MobiC to $17.5 \%$ for the Secure-C to $14.7 \%$ for the Prodisc C, while for the Prestige LP, disk pressures actually increased by $4.6 \%$ compared to the intact spinal column.

\section{Facet force responses under hybrid loading}

Fig. 7 shows changes in the facet forces responses of the four CDAs concerning the intact spine in extension at all levels. At the rostral segment, facet loads ranged from $76 \%$ to $83 \%$ of the intact spine for all disks and at the caudal segment, the outcome was similar (range, $78 \%$ to $87 \%$ ). At the index level, they increased by 2.04 to 3.20 times, with the smallest increase in the Secure- $\mathrm{C}$ and the highest increase in the Mobi-C device.

\section{Discussion}

\section{Rationale for the modeling aspects of our study design}

The objective of this research was to evaluate the responses of FDA approved CDAs for single-level arthroplasty. This study was initiated by a need to compare the biomechanical responses of four different types (constraint, design, and material) of CDAs directly, using a model with full repeatability and not confounded by normal biological variabilities that exist with in vivo human and human cadaver studies. While clinical studies provide information on the outcomes, such as heterotopic ossifications and

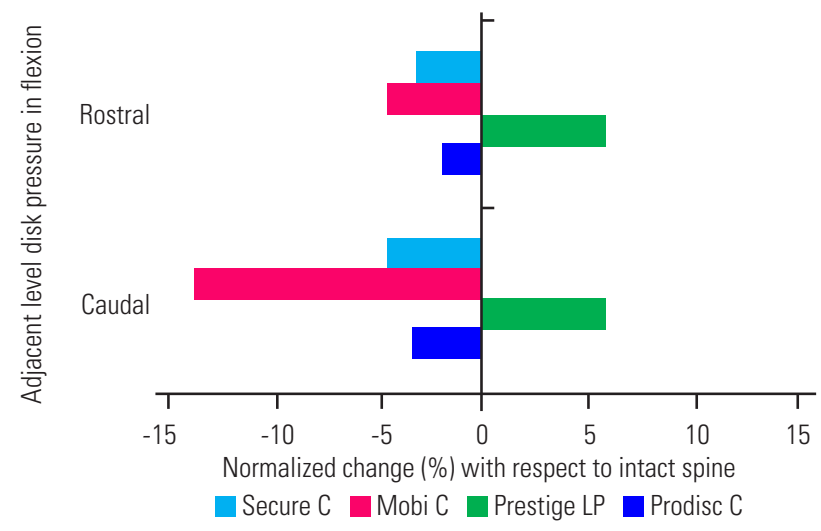

Fig. 5. Bar chart showing the normalized disk pressures under flexion for different cervical disk arthroplasties.

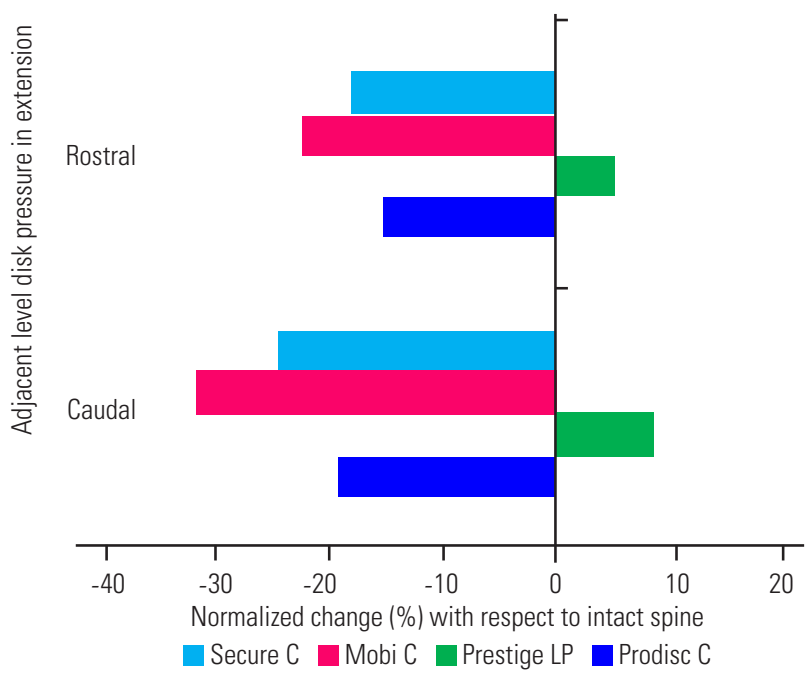

Fig. 6. Bar chart showing the normalized disk pressures under extension for different cervical disk arthroplasties.

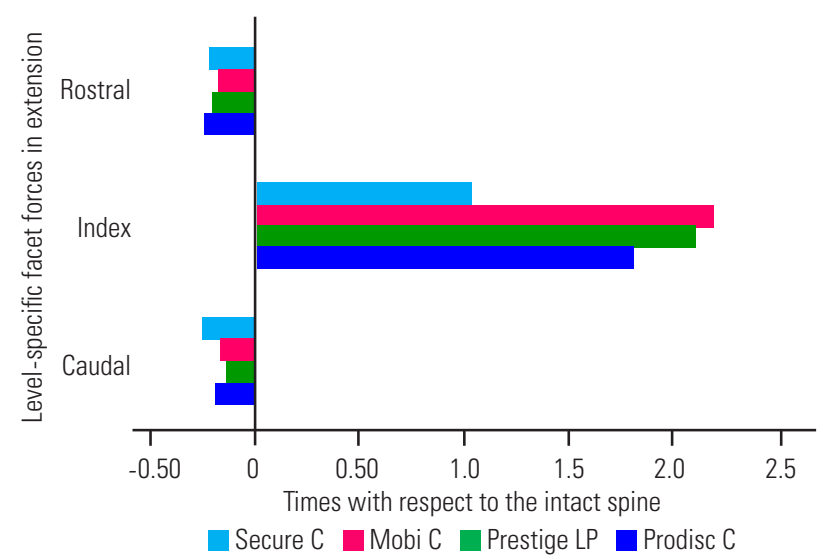

Fig. 7. Bar chart showing the increased facet loads with respect to the intact spine for different cervical disk arthroplasties. 
ASD, segment/level-specific responses at the local component level in the form of disk pressures and facet loads (bicolumn load-sharing biomechanics) cannot be extracted from patient images or response questionnaires. Likewise, human cadaver models also are relatively ineffective because it is necessary to introduce sensors (pressure gages and load cells) to obtain bi-column data. Implanting load cells compromise joint integrity, and also act as a secondary load path because load cells are more rigid than the joint into which they are introduced. Furthermore, the biological variability associated with cadavers makes it difficult to compare the four CDAs without large sample sizes and appropriately accounting for the inherent spine morphologies. Finite element models can be exercised repeatedly with full reproducibility and implantation of various devices, and these considerations were used in the current study of CDAs.

\section{Background for selection of devices}

The criteria for choosing the specific devices were as follows: (1) approval of the FDA for human implantation, i.e., a device that is not currently undergoing clinical trials; (2) approval for inserting the device to any segment of the cervical spine; and (3) device types/features included their constraining nature, implant material, and design. The Prestige LP is an unconstrained device. The Prodisc C, Mobi- $\mathrm{C}$, and Secure- $\mathrm{C}$ are considered semi-constrained devices. While the Mobi-C and Secure- $\mathrm{C}$ are triple-component devices, the other two are double-component devices. The Prestige LP is metal-on-metal, while the other three are metal on polyethylene. Out of the four devices, the Mobi-C and Prestige LP are approved by the FDA for bi-level fusions. Because the other two are approved for only single-level fusion, a single implant level was selected to compare the results of each prosthesis properly. Also, the study simulated surgery at C5-C6 because it is the most commonly involved level. It would be, however, a relatively straightforward exercise to simulate other types, e.g., hybrid surgeries and bi-level CDA options, and this will be considered for a future investigation topic.

\section{Loading and output parameters}

Flexion and extension bending moments were applied to the cervical spine for the analysis of the four CDAs. The procedure followed the conventional methods, i.e., apply a pure moment loading to the intact spine, in both modalities. The overall ROM of the spinal column was calculated. Then, we determined the bending moment in both modes to each CDA spine that matched the ROM determined in the pure moment condition for the intact spine. In other words, the hybrid moment protocol was followed in the present study, a protocol also used in other studies [18-21]. While CDAs are used in the military, impact loads should be studied because the population is subjected to activities involving higher g-forces, routinely $[22,23]$. Current, results of angular motion and bi-column load-sharing at the rostral and caudal segments, and motion and facet forces at the index level, under clinically relevant loading modes, form a comparison of the basic external and intrinsic biomechanical responses of the spine with the four CDAs. To our knowledge, this type of analytical comparison has not been reported in the literature for these devices. As expected, variations, and similarities in the biomechanical parameters stem from individual characteristics of the $\mathrm{CDA}$ itself. Acknowledging that any CDA's ultimate goal is to improve the patient's quality of life and act as a superior alternative to conventional $\mathrm{ACDF}$, in what follows, these responses are interpreted in light of their design differences $[1,4,6,24,25]$.

The hybrid moments were highest for the Prestige LP disk because of its metal to metal contact design, in contrast to the other CDAs containing an intervening polymer. This was true in both extension and flexion moments. Lower bending moments $(<2 \mathrm{Nm})$ are required to achieve the motion-controlled hybrid protocol magnitudes. Matching the overall column ROM for both pure moment and hybrid protocols, for the Secure-C, Prodisc C, and Mobi-C will reflect the relative softness of these three CDAs as compared to the Prestige LP. The Prestige LP device required a higher magnitude $(2.3 \mathrm{Nm}$ for flexion and $2.2 \mathrm{Nm}$ for extension). This is expected because of design differences, i.e., the metal to polymer versus metal to metal interaction. These design differences, as discussed below, contributed to the angular kinematics of the index, rostral and caudal levels, and the bi-column load paths.

The present study was focused on motion in the sagittal plane, as flexion-extension is common in day to day activities, while the other modes also exist. Specifically, concerning the cervical spine, motions are more coupled between the axial rotation and lateral bending modalities $[26,27]$. With flexion-extension, however, such coupling is limited, and this was the primary reason for choosing this 
mode. While finite element analysis is useful to study the response under axial rotation and lateral bending modes, they are to be considered for future investigation.

\section{Cervical disk arthroplasty-specific responses}

\section{1) Prestige LP}

The most considerable change was the increased motion at the index level under extension (26\%), followed by flexion (15\%); however, the increase in the facet load-sharing (3.1 times intact spine) at the index level was considerable. These results suggest that the implant's metal-on-metal design is effective in increasing/preserving index level mobility. However, it places an enhanced demand on the posterior structures. The peak effect on the adjacent levels was a $12 \%$ decrease in extension motion at the rostral segment and a similar reduction at the caudal segment in flexion. The $8 \%$ increase in disk pressure at the caudal segment under extension is somewhat minimal (less than $10 \%)$, while the facet load-sharing decreased slightly in both adjacent levels (approximately 15\%). These findings further suggest that this CDA also decreases the motion and posterior column loads at adjacent levels, implying its efficacy under sagittal moment loading conditions.

\section{2) Prodisc $C$}

The most considerable change was in the increased motion at the index level under extension (36\%), followed by flexion (15\%); however, the increase in the facet loadsharing (2.8 times intact spine) at the index level was considerable. The peak effect on the adjacent level was a $19 \%$ decrease in extension motion at the rostral segment and minimal changes in the disk pressures and facet loads. These results suggest that this CDA's metal-polyethylene design considerably increases index level mobility and reduces rostral segment mobility, and changes in the bicolumn load-sharing are insignificant. Any potential implications due to the sagittal moment loads may be biased at the rostral segment.

\section{3) Mobi-C}

The changes in increased ROM at the index level of $37 \%$ and $32 \%$ under extension and flexion, and facet loadsharing (3.1 times intact spine) were considerable. The peak effects on the adjacent level were $17 \%$ and $12 \%$ decreases in extension motion at the rostral and flexion motion at the caudal segments, and $22 \%$ and $14 \%$ decrease in disk pressures at the rostral segment under extension and caudal segment under flexion. The changes in the facet loads were, however, minimal. These results suggest that the implant's metal-polyethylene design changes all three divisions of the spine with emphasis on the anterior column, i.e., disks.

\section{4) Secure-C}

The changes in increased ROM at the index level of $48 \%$ and $24 \%$ under extension and flexion, and facet loadsharing (twice intact spine) were considerable. The peak effects on the adjacent level were $16 \%$ decreased motion under extension at the rostral segment, and $24 \%$ and $17 \%$ decrease in disk pressure at the caudal and rostral segments under extension with minimal changes in the facet loads. These results suggest that the implant's metal-polyethylene design changes all three divisions of the spine, with emphasis on the anterior column.

\section{Evaluations of cervical disk arthroplasty responses}

The most considerable change in the index level kinematics of all devices was in extension, although the changes were symmetrical with the Mobi-C. The minimal changes with the Prestige LP are due to the two-part and metalon-metal design that is in contrast to the metal on polyethylene components of the other three CDAs. The most considerable changes for the Mobi- $\mathrm{C}$ and Secure- $\mathrm{C}$ devices are expected because they are of metal-polyethylene designs that allow translation $(1.0$ and $1.25 \mathrm{~mm}$, respectively) and both allow more than $10^{\circ}$ of sagittal motion. The greater increase in Secure-C, of $48 \%$, may be due to the three moving components, i.e., sliding core with spherical superior and cylindrical inferior cores, while the relatively smaller increase in Mobi-C (37\%) is attributed to the partial surface area covered by the softer mobile dome core.

Although the facet column load-sharing increased at the index level in all CDAs (2 to 3 times more as compared to the intact spine), the smallest increase occurred in the Secure- $\mathrm{C}$ device. The sliding core may have acted as a shield as compared to the other three devices that had more substantial increases in posterior column loading. Since increased load-sharing may suggest increased demand over time, these results indicate the need to focus on facet-related issues arising from flexion-extension loading in the in vivo environment. 
At the adjacent levels, motion decreases for all devices underscore the efficacy of CDA as compared to conventional ADCF. The greater decreases in extension versus flexion for the rostral segment in all devices suggest increased stiffness under this mode. The Prestige LP responded with the least change due to its metal-on-metal design, and this was true for both extension and flexion modes. While the disk pressure changes were minimal in flexion for all disks (less than 10\%, except for the Mobi-C caudal segment), under extension, the pressure decreased further in the Mobi-C and Secure-C disks, while showing minimal increases in the other two devices. The threepiece design of these two devices may have contributed to this phenomenon. The minimal changes in the facet loads for all disks at both adjacent levels are suggestive of their lesser role in influencing the adjacent posterior column biomechanics.

\section{Clinical relevance}

Our study validates clinical observations and criteria applicable to CDA. Increased ROM and facet loading at the index level in all four artificial disks support contraindications for arthroplasty: facet arthroplasty and instability. Improper anchoring of the implants (mechanical keel design or osteointegration) may lead to migration. Our study validates one of the primary reasons for performing arthroplasty, i.e., minimizing ASD while preserving motion at the index level. This is clinically important as ASD can lead to future surgery. It is also essential for patient education when obtaining consent for choosing CDA over ACDF.

Our study quantifies the reduction in ROM and facet loading at the adjacent levels and provides objective data as to how these disk prostheses differ in reducing ASD, via quantification of intradiscal pressures. The reduction in the pressure in the Bryan, Mobi-C, and Prodisc $\mathrm{C}$ devices, as compared to the pressure increase found with the Prestige LP, demonstrates the artificial disk design's role on the biomechanical responses of the cervical spine. The metalon-metal design of the Prestige LP resulted in this unique finding as compared to the polymer-integrated designs. This is clinically important because the adjacent disks may be at a higher risk of degeneration with the metal-on-metal device, as compared to other artificial disks. In an era of the increasing popularity of arthroplasty, spinal surgeons need to understand the differing biomechanical responses of various artificial disks. The direct clinical implications should be discussed with the patient preoperatively.

\section{Limitations}

While differences in the responses were evident between the four CDAs, the minimum percentage difference in the outcome measures considered significant is more involved. This is because the CDA's performance depends on the longitudinal and occupation-related exposures of a specific patient, issues that were not considered herein. Military patients are generally younger and healthier than civilian patients, while at the same time; military patients are exposed to more demanding loading situations. The minimum percentage differences are, therefore, expected to vary between the two populations, with a likely trend toward a larger difference for the civilian application. Future studies should include consideration of these factors.

Our study, focusing on the biomechanical response evaluations of the four CDAs under physiological loadings, has significant clinical relevance. Mechanical loading is a day to day event regardless of the status of the spine, be it healthy, diseased, or CDA-implanted, as well as patient occupation and demographics. As CDA is used commonly in younger patients, an understanding of the biomechanics is even more critical because loading effects prevail for a longer life span. This is particularly true in the military, as CDA procedures are performed to expedite and facilitate an early return to duty. The soldiers' operational activities involve higher g-forces. For example, wearing a helmet increases the axial load on the cervical spine. It is critical to understand the responses of different CDAs due to other types of mechanical loads. This study focused on single-cycle loading, while the CDA's effects on patient outcomes over time are longitudinal. Cyclic loading requires consideration. Heterotopic ossification is also an important issue because any osseous growth around the implant compromises the original intent, in that motion preservation will not be optimally achieved, and ASD paralleling the ACDF procedure may be a result. The authors are pursuing such topics with finite element and experimental models to address these issues.

\section{Conclusions}

While all of the artificial cervical disks restored motion at the index level, differences existed between CDAs regard- 
ing facet column load-sharing. Both intradiscal pressures and facet column load-sharing depended on the type of the CDA. Consideration of facet joint integrity should be a factor in the clinical decision-making process for selecting the CDA. The metal-on-metal disk is different from the metal on polymer designs, and factors such as the allowance of anteroposterior translation are attributable to these differences. The clinical relevance of these findings is that they are helpful to the spinal surgeon in both decision-making and patient education.

\section{Conflict of Interest}

No potential conflict of interest relevant to this article was reported.

\section{Acknowledgments}

This study was supported in part by the Office of the Assistant Secretary of Defense for Health Affairs, through the Broad Agency Announcement under Award No. W81XWH-16-1-0010. NY and SK are employees of the Zablocki VA Medical Center, Milwaukee, WI, USA. The Opinions, interpretations, conclusions, and recommendations are those of the authors and are not necessarily endorsed by the US Department of Defense or other sponsors.

\section{Author Contributions}

Data acquisition: YP; analysis of data: YP, HC, NY, DJ; drafting of the manuscript: YP, HC, NY, DJ, JB; conception and design: HC, NY, JB; critical revision: HC, NY, SK; administrative support: NY, SK; and supervision: NY, DJ, SK.

\section{References}

1. Smith GW, Robinson RA. The treatment of certain cervical-spine disorders by anterior removal of the intervertebral disc and interbody fusion. J Bone Joint Surg Am 1958;40-A:607-24.

2. Hilibrand AS, Carlson GD, Palumbo MA, Jones PK, Bohlman HH. Radiculopathy and myelopathy at segments adjacent to the site of a previous anterior cervical arthrodesis. J Bone Joint Surg Am 1999;81:51928.
3. Hilibrand AS, Robbins M. Adjacent segment degeneration and adjacent segment disease: the consequences of spinal fusion? Spine J 2004;4(6 Suppl):190S-194S.

4. Rao RD, Gore DR, Tang SJ, Rebholz BJ, Yoganandan $\mathrm{N}$, Wang M. Radiographic changes in the cervical spine following anterior arthrodesis: a longterm analysis of 166 patients. J Bone Joint Surg Am 2016;98:1606-13.

5. Burkus JK, Traynelis VC, Haid RW Jr, Mummaneni PV. Clinical and radiographic analysis of an artificial cervical disc: 7-year follow-up from the Prestige prospective randomized controlled clinical trial: clinical article. J Neurosurg Spine 2014;21:516-28.

6. Alvin MD, Abbott EE, Lubelski D, et al. Cervical arthroplasty: a critical review of the literature. Spine J 2014;14:2231-45.

7. Gornet MF, Burkus JK, Shaffrey ME, Argires PJ, Nian H, Harrell FE Jr. Cervical disc arthroplasty with PRESTIGE LP disc versus anterior cervical discectomy and fusion: a prospective, multicenter investigational device exemption study. J Neurosurg Spine 2015;23:558-73.

8. John JD, Arun MW, Yoganandan N, Kurpad SN, Gurunathan SK. Mapping block-based morphing for subject-specific spine finite element models. Proceedings of the 54th Annual Rocky Mountain Bioengineering Symposium; 2017 Mar 31-Apr 1; Denver, USA. Durham (NC): International Society of Automation' 2017.

9. Choi H, Purushothaman Y, Baisden J, Yoganandan N. Unique biomechanical signatures of Bryan, Prodisc C, and Prestige LP cervical disc replacements: a finite element modelling study. Eur Spine J 2019 Oct 12 [Epub]. https://doi.org/10.1007/s00586-019-06113-y.

10. John JD, Saravana Kumar G, Yoganandan N. Cervical spine morphology and ligament property variations: a finite element study of their influence on sagittal bending characteristics. J Biomech 2019;85:18-26.

11. Wheeldon JA, Stemper BD, Yoganandan N, Pintar FA. Validation of a finite element model of the young normal lower cervical spine. Ann Biomed Eng 2008;36:1458-69.

12. Wheeldon JA, Pintar FA, Knowles S, Yoganandan N. Experimental flexion/extension data corridors for validation of finite element models of the young, normal cervical spine. J Biomech 2006;39:375-80. 
13. Mercer S, Bogduk N. The ligaments and annulus fibrosus of human adult cervical intervertebral discs. Spine (Phila Pa 1976) 1999;24:619-28.

14. Kumaresan S, Yoganandan N, Pintar FA. Finite element analysis of the cervical spine: a material property sensitivity study. Clin Biomech (Bristol, Avon) 1999;14:41-53.

15. Lu H, Peng L. Efficacy and safety of Mobi-C cervical artificial disc versus anterior discectomy and fusion in patients with symptomatic degenerative disc disease: a meta-analysis. Medicine (Baltimore) 2017;96:e8504.

16. Gornet MF, Lanman TH, Burkus JK, et al. Cervical disc arthroplasty with the Prestige LP disc versus anterior cervical discectomy and fusion, at 2 levels: results of a prospective, multicenter randomized controlled clinical trial at 24 months. J Neurosurg Spine 2017;26:653-67.

17. Cusick JF, Pintar FA, Yoganandan N. Biomechanical alterations induced by multilevel cervical laminectomy. Spine (Phila Pa 1976) 1995;20:2392-9.

18. Gandhi AA, Kode S, DeVries NA, Grosland NM, Smucker JD, Fredericks DC. Biomechanical analysis of cervical disc replacement and fusion using single level, two level, and hybrid constructs. Spine (Phila Pa 1976) 2015;40:1578-85.

19. Liao Z, Fogel GR, Wei N, Gu H, Liu W. Biomechanics of artificial disc replacements adjacent to a 2-level fusion in 4-level hybrid constructs: an in vitro investigation. Med Sci Monit 2015;21:4006-14.

20. Panjabi M, Malcolmson G, Teng E, Tominaga Y, Henderson G, Serhan H. Hybrid testing of lumbar
CHARITE discs versus fusions. Spine (Phila Pa 1976) 2007;32:959-67.

21. Chen WM, Jin J, Park T, Ryu KS, Lee SJ. Strain behavior of malaligned cervical spine implanted with metal-on-polyethylene, metal-on-metal, and elastomeric artificial disc prostheses: a finite element analysis. Clin Biomech (Bristol, Avon) 2018;59:19-26.

22. Yoganandan N, Pintar FA, Kleinberger M. Whiplash injury: biomechanical experimentation. Spine (Phila Pa 1976) 1999;24:83-5.

23. Yoganandan N, Pintar FA, Sances A Jr, Reinartz J, Larson SJ. Strength and kinematic response of dynamic cervical spine injuries. Spine (Phila Pa 1976) 1991;16(10 Suppl):S511-7.

24. Dufour T, Beaurain J, Huppert J, Dam-Hieu P, Bernard P, Steib JP. Clinical and radiological evaluation of cervical disc arthroplasty with 5-year followup: a prospective study of 384 patients. Eur Spine J 2019;28:2371-9.

25. Nunley PD, Coric D, Frank KA, Stone MB. Cervical disc arthroplasty: current evidence and real-world application. Neurosurgery 2018;83:1087-106.

26. Yoganandan N, Pintar FA, Stemper BD, Wolfla CE, Shender BS, Paskoff G. Level-dependent coronal and axial moment-rotation corridors of degeneration-free cervical spines in lateral flexion. J Bone Joint Surg Am 2007;89:1066-74.

27. Yoganandan N, Stemper BD, Pintar FA, Baisden JL, Shender BS, Paskoff G. Normative segment-specific axial and coronal angulation corridors of subaxial cervical column in axial rotation. Spine (Phila Pa 1976) 2008;33:490-6. 